\title{
Revealing hidden supercooled liquid states in Al-based metallic glasses by ultrafast scanning calorimetry: Approaching theoretical ceiling of liquid fragility
}

\author{
Qun Yang ${ }^{1}$, Jing Huang ${ }^{2}$, Xiao-Hui Qin ${ }^{1}, \mathrm{Fa}-\mathrm{Xi} \mathrm{Ge}^{1}$ and Hai-Bin Yu ${ }^{1 *}$
}

Metallic glasses (MGs) are amorphous alloys with a number of unique properties that are attractive for the fundamental understanding of the nature and applications of disordered systems [1-17]. Generally, MGs might be grouped into two categories based on their glass forming ability (i.e., the ease of glass formation by cooling a liquid): in one case, large or bulk volumes may be slowly cooled to the glassy state from the melt. This category is usually called as bulk metallic glass formers [18-22]. In contrast, certain MGs, represented by aluminum (Al)$[23,24]$ and some iron (Fe)- $[25,26]$ based MGs, can be synthesized mainly by rapid solidification processes such as melt spinning or vapor deposition. These MGs are often denoted as marginal MG-formers [23-25].

With the rapid developments of MGs, many previously identified marginal glass formers have been successfully made into bulk metallic glasses by composition strategies (such as Fe- and Ni-based MGs) [8,25,26,28]. However, one remarkable exception is the family of Al-based MGs $[23,24]$. Although there have been continuous efforts in optimizing compositions and improving processing techniques since its first discovery [29] several decades ago, the glass forming ability of Al-MGs is still limited nowadays [23,30,31]. Recently, Wu et al. [30] reported that the hitherto best glass forming ability for Al-MGs is from $\mathrm{Al}_{86} \mathrm{Ni}_{6.75} \mathrm{Co}_{2.25} \mathrm{Y}_{3.25} \mathrm{La}_{1.75}$ with a fully glassy rod of $1.5 \mathrm{~mm}$ in diameter. Although the glassy rod can reach $2.5 \mathrm{~mm}$ for the same composition after a refined fluxtreatment of the liquid [31], this critical size is still much smaller than those of typical Zr-based MGs $(\sim 75 \mathrm{~mm})$ [32], Mg-MGs (25 mm) [18] and Pd-MGs (80 mm) [33]. Moreover, the glass forming ability of Al-MGs is very sensitive to compositions. Only a few percent change of the constituting elements would dramatically reduce this critical size $[21,27,30,34,35]$. Consequently, it is more difficult to develop Al-MGs than other MGs. Fundamentally, it remains to be a puzzling issue why the AlMGs are so unique $[27,30,36]$.

Among several factors, one clue for the distinction between bulk glasses and marginal glass formers is based upon their supercooled liquids $[19,23,26,27]$. The change of the relaxation dynamics (e.g., viscosity, diffusivity or relaxation time) with the temperature is highly materialspecific. Angell introduced the concept of fragility $(m)$ for the classification of glass-forming materials, which was defined as the apparent activation energy of relaxation time $\tau_{\alpha}$ normalized to glass transition temperature $T_{\mathrm{g}}$ : $m=\mathrm{d} \log \left(\tau_{\alpha}\right) /\left.\left(\mathrm{d} T_{\mathrm{g}} / T\right)\right|_{T=T_{\mathrm{g}}}$ [37]. A stronger deviation from Arrhenius behavior (with a larger $m$ value) corresponds to a more fragile system; otherwise, the system is strong [37-40]. Wang et al. [41] theoretically pointed out that the fragility index for non-polymeric glasses should have a lower limit $m=16.5$, and an upper limit $m \sim 175$.

Recently, Johnson et al. [42] conducted a critical assessment of the database of metallic glasses, and they proposed that the glass forming ability can be expressed by a two-variable empirical function based on $m$ and $T_{\mathrm{rg}}$ ( $T_{\mathrm{rg}}$ is the reduced $T_{\mathrm{g}}$ ). The larger values of $T_{\mathrm{g}} / T_{\mathrm{m}}$ and the smaller values of $m$ are favored by stronger glass formation. Their findings highlight the important role of liquid dynamics on the glass formation.

However, until now, almost none of the melt-quenched Al-MGs exhibits recognizable glass transition or the supercooled liquid region when measured by the differential

${ }^{1}$ Wuhan National High Magnetic Field Center and School of Physics, Huazhong University of Science and Technology, WuHan 430074, China

${ }^{2}$ School of Materials and Engineering, Huazhong University of Science and Technology, Wuhan 430074, China

* Corresponding author (email: habinyu@hust.edu.cn) 
scanning calorimetry (DSC) $[23,27,30,43]$. Instead, they show an exothermic process due to the primary crystalline phase formation of aluminum [23,27,30]. Hence, properties of the supercooled liquid and especially the fragility of Al-MGs are still unknown. In a previous work, Hu et al. [44] inferred that the Al-MGs might have large values of $m$. However, as the glass transition of Al-MGs could not be probed by DSC, they used the crystallization process as an estimation of the glass transition.

In this work, utilizing the recently developed fast scanning calorimetry (FSC) [45-55] to bypass the primarily crystallization process, we present the direct characterization of the glass transition and supercooled liquid states of 20 different Al-based MGs with different compositions and glass forming ability. The results indicate that the Al-MGs feature remarkably high values of fragility, which are sensitive to minor compositional adjustment. Some of them almost reach the theoretical upper limit of fragility [41]. The lowest fragility in these studied Al-MGs is found in the $\mathrm{Al}_{86} \mathrm{Ni}_{6.75} \mathrm{Co}_{2.25} \mathrm{Y}_{3.25} \mathrm{La}_{1.75}$ MG which also has the best glass forming ability. Our findings suggest that the highly fragile nature of the su- percooled liquids could be a common controlling factor for the marginal glass forming ability of Al-MGs. Thus, strategies for reducing fragility could be taken into considerations in enhancing glass forming ability of Al-MGs. Meanwhile, the Al-MGs provide a benchmark model for the understanding of the origin of fragility in glasses.

We investigated 20 different Al-MGs as listed in Table 1 by melt-spinning techniques. First, ingots of the Al-based alloy were prepared by melting high purity elements (purity $\geq 99.99$ at.\%) under a Ti-gettered argon atmosphere in an arc-melting furnace. The ingots were re-melted five times to ensure homogeneity. Then, amorphous ribbons of different thicknesses were achieved by varying the rotational speed $\left(10-60 \mathrm{~m} \mathrm{~s}^{-1}\right)$ of the rollers. The glass forming ability was characterized by the critical thickness at which fully amorphous materials can be obtained. X-ray diffraction (XRD, Bruke D2 phaser) and DSC were used to check the amorphous nature of these ribbons.

The glass transition and crystallization of alloys at relatively high heating rates were obtained by continuous heating on a Flash DSC (Mettler Toledo FSC 2+) under

Table 1 Compositions of Al-MGs studied in this work and their $T_{\mathrm{g}}, T_{\mathrm{x}}$ and $\Delta T_{\mathrm{x}}$ (measured at $\left.600 \mathrm{~K} \mathrm{~s}{ }^{-1}\right), T_{\mathrm{m}}, T_{\mathrm{g} 20}, h_{\mathrm{c}}$, and $m$ ( \pm 5 error bar). $T_{\mathrm{g} 20}$ is the extrapolated $T_{\mathrm{g}}$ at a heating rate of $20 \mathrm{~K} \mathrm{~min}^{-1}$ (which is commonly used in glass physics). $h_{\mathrm{c}}( \pm 5 \mu \mathrm{m}$ error bar) is the critical thickness of glass ribbon which is fully amorphous.

\begin{tabular}{|c|c|c|c|c|c|c|c|c|}
\hline & Composition & $T_{\mathrm{g}}(\mathrm{K})$ & $T_{\mathrm{x}}(\mathrm{K})$ & $\Delta T_{\mathrm{x}}(\mathrm{K})$ & $T_{\mathrm{m}}(\mathrm{K})$ & $T_{\mathrm{g} 20}(\mathrm{~K})$ & $h_{\mathrm{c}}(\mu \mathrm{m})$ & $m$ \\
\hline 1 & $\mathrm{Al}_{84} \mathrm{Ni}_{10} \mathrm{Ce}_{6}$ & 564.5 & 629.7 & 65.2 & 892.3 & 529.6 & 60 & 107 \\
\hline 2 & $\mathrm{Al}_{86} \mathrm{Ni}_{9} \mathrm{Ce}_{5}$ & 556.2 & 621.6 & 65.4 & 890.6 & 525.7 & 60 & 129 \\
\hline 3 & $\mathrm{Al}_{86} \mathrm{Ni}_{6.75} \mathrm{Co}_{2.25} \mathrm{Ce}_{5}$ & 556.2 & 611.8 & 55.6 & 889.4 & 526.8 & 50 & 164 \\
\hline 4 & $\mathrm{Al}_{86} \mathrm{Ni}_{4} \mathrm{Co}_{5} \mathrm{Ce}_{5}$ & 581.9 & 621.5 & 39.6 & 897.9 & 547.8 & 40 & 120 \\
\hline 5 & $\mathrm{Al}_{86} \mathrm{Co}_{9} \mathrm{Ce}_{5}$ & 633.2 & 662.1 & 28.9 & 903.1 & 593.8 & 20 & 127 \\
\hline 6 & $\mathrm{Al}_{86} \mathrm{Ni}_{9} \mathrm{La}_{5}$ & 595.7 & 658.0 & 62.3 & 890.5 & 561.3 & 40 & 119 \\
\hline 7 & $\mathrm{Al}_{86} \mathrm{Ni}_{6.75} \mathrm{Co}_{2.25} \mathrm{La}_{5}$ & 551.7 & 609.5 & 57.8 & 893.7 & 526.3 & 40 & 164 \\
\hline 8 & $\mathrm{Al}_{86} \mathrm{Ni}_{4} \mathrm{Co}_{5} \mathrm{La}_{5}$ & 591.5 & 640.8 & 49.3 & 900.0 & 558.3 & 30 & 117 \\
\hline 9 & $\mathrm{Al}_{85} \mathrm{Ni}_{10} \mathrm{Sm}_{5}$ & 539.6 & 607.8 & 68.2 & 897.9 & 510.4 & 50 & 146 \\
\hline 10 & $\mathrm{Al}_{85} \mathrm{Ni}_{10} \mathrm{Ce}_{1.75} \mathrm{Sm}_{3.25}$ & 541.4 & 607.6 & 66.2 & 895.8 & 514.8 & 140 & 122 \\
\hline 11 & $\mathrm{Al}_{85} \mathrm{Ni}_{10} \mathrm{Ce}_{3.25} \mathrm{Sm}_{1.75}$ & 547.0 & 608.4 & 61.4 & 889.4 & 512.0 & 110 & 104 \\
\hline 12 & $\mathrm{Al}_{85} \mathrm{Ni}_{10} \mathrm{Ce}_{5}$ & 549.7 & 612.6 & 62.9 & 892.6 & 519.7 & 80 & 132 \\
\hline 13 & $\mathrm{Al}_{84} \mathrm{Ni}_{11} \mathrm{Sm}_{5}$ & 580.1 & 646.8 & 66.7 & 897.9 & 540.2 & 70 & 102 \\
\hline 14 & $\mathrm{Al}_{86} \mathrm{Ni}_{9} \mathrm{Sm}_{5}$ & 522.3 & 566.7 & 44.4 & 899.7 & 491.9 & 90 & 128 \\
\hline 15 & $\mathrm{Al}_{87} \mathrm{Ni}_{8} \mathrm{Sm}_{5}$ & 532.8 & 584.6 & 51.8 & 895.8 & 498.6 & 50 & 107 \\
\hline 16 & $\mathrm{Al}_{90} \mathrm{Sm}_{10}$ & 531.6 & 563.1 & 31.5 & 906.4 & 505.2 & 20 & 162 \\
\hline 17 & $\mathrm{Al}_{90} \mathrm{Ce}_{10}{ }^{\mathrm{a}}$ & & & & 909.6 & & 10 & \\
\hline 18 & $\mathrm{Al}_{86} \mathrm{Ni}_{6.75} \mathrm{Co}_{2.25} \mathrm{Sm}_{3.25} \mathrm{La}_{1.75}$ & 549.0 & 606.3 & 57.3 & 889.4 & 513.6 & 90 & 111 \\
\hline 19 & $\mathrm{Al}_{86} \mathrm{Ni}_{6.75} \mathrm{Co}_{2.25} \mathrm{Sm}_{3.25} \mathrm{Ce}_{1.75}$ & 546.1 & 602.9 & 56.8 & 889.4 & 516.2 & 50 & 120 \\
\hline 20 & $\mathrm{Al}_{86} \mathrm{Ni}_{6.75} \mathrm{Co}_{2.25} \mathrm{Y}_{3.25} \mathrm{La}_{1.75}$ & 543.2 & 598.5 & 55.3 & 896.8 & 503.7 & 170 & 96 \\
\hline
\end{tabular}

a) The glass transition could not be probed by FSC even with the maximum heating rate of $15,000 \mathrm{~K} \mathrm{~s}^{-1}$. 
$80 \mathrm{~mL} \mathrm{~min}^{-1}$ argon gas flow. The twin-type chip sensor based on MEMS technology was made with a sample and a reference side. Samples were placed on the sensitive area of a MultiSTAR UFS 1 MEMS chip sensor in a range of heating rates from 5 to $15,000 \mathrm{~K} \mathrm{~s}^{-1}$. In order to ensure the reliability of the measurement, each crystallized sample was heated again to obtain a baseline. The crystal melting temperature and the liquid temperature were measured using a differential thermal analysis (TA SDT Q600) instrument at a heating rate of $20 \mathrm{~K} \mathrm{~min}^{-1}$ under a $100 \mathrm{~mL} \mathrm{~min}^{-1}$ argon gas flow. These samples were also measured at relatively low heating rates by a conventional DSC (Mettler Toledo DSC 3) under a $50 \mathrm{~mL} \mathrm{~min}^{-1} \mathrm{ni}-$ trogen gas flow. The conventional DSC was calibrated by using pure In and $\mathrm{Zn}$ as standard references.

Fig. 1a compares two typical heat flow curves of an $\mathrm{Al}_{85} \mathrm{Ni}_{10} \mathrm{Ce}_{5}$ MG measured by a conventional DSC (at a heating rate $Q=0.333 \mathrm{~K} \mathrm{~s}^{-1}$ or $20 \mathrm{~K} \mathrm{~min}^{-1}$ ) and a FSC $\left(Q=600 \mathrm{~K} \mathrm{~s}^{-1}\right)$, respectively. The DSC curve shows only an unobvious signature of glass transition onset which is truncated by a sharp exothermic peak of the primary crystallization. In contrast, the FSC curve exhibits a clear glass transition $\left(T_{\mathrm{g}}=549.7 \mathrm{~K}\right.$ at this heating rate). The first crystallization peak has been shifted to a higher temperature $\left(T_{\mathrm{x}}=612.6 \mathrm{~K}\right.$, where $T_{\mathrm{x}}$ is the first crystallization temperature) by the rapid heating. The temperature difference between $T_{\mathrm{x}}$ and $T_{\mathrm{g}}$ defines the supercooled liquid region $\Delta T_{\mathrm{x}}=63 \mathrm{~K}$ at this heating rate.
Thus, the FSC provides us a unique means to characterize the supercooled liquid states of Al-MGs.

Fig. 1b shows a set of typical FSC measurements over a wide range of heating rates, ranging from $Q=10$ to $4500 \mathrm{~K} \mathrm{~s}^{-1}$. We find that only for $Q \geq 30 \mathrm{~K} \mathrm{~s}^{-1}$, the glass transition can be fully resolved by our FSC measurements. This critical heating rate is much higher than the commonly used DSC $\left(0.1-1 \mathrm{~K} \mathrm{~s}^{-1}\right)$. The inset of Fig. 1c summarizes the primary (first) crystallization peak temperature both from DSC and FSC at different heating rates, in terms of the so-called Kissinger plot [38]. It shows that the two sets of data (DSC and FSC) match with each other consistently. This suggests that the primary crystallization on the FSC might be the same process as DSC but delay to a higher temperature by the fast heating.

We determined the fragility index $m$ based on the heating rate dependence of $T_{\mathrm{g}}$, as shown in Fig. 1c. By fitting these data with the Vogel-Fulcher-Tammann (VFT) equation, we calculated $m=132 \pm 3$ for the $\mathrm{Al}_{85} \mathrm{Ni}_{10} \mathrm{Ce}_{5} \mathrm{MG}$, which implied a rather fragile behavior. In the Supplementary information, we validated our approach by comparing the fragility for a $\mathrm{Pd}_{40} \mathrm{Ni}_{30} \mathrm{Cu}_{10} \mathrm{P}_{10}$ MG between known fragility $(m=52 \pm 2)$ from previous viscosity measurement [56] and current method ( $m=55$ \pm 3 , Fig. S1a). In a previous work, Evenson et al. [57] illustrated that by heating and cooling a glass to the supercooled liquid state at the same rate would result in a
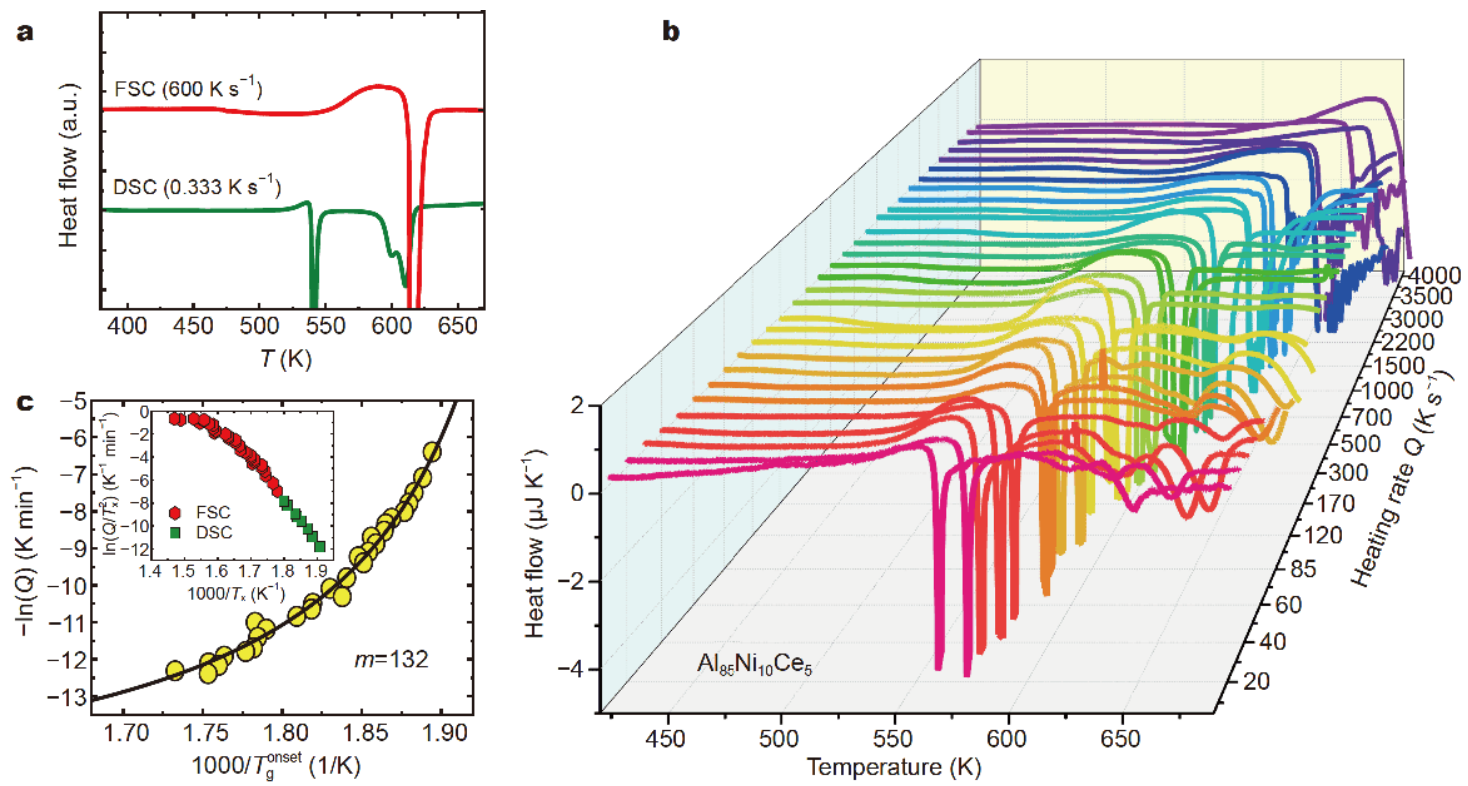

Figure 1 Typical scanning calorimetry for $\mathrm{Al}_{85} \mathrm{Ni}_{10} \mathrm{Ce}_{5} \mathrm{MG}$. (a) Comparative studies between low heating rate (conventional DSC) and high heating rate (FSC). (b) Heat flow curves measured by FSC using different heating rates. (c) The onset temperature of glass transition, $T_{\mathrm{g}}$ onset, against the

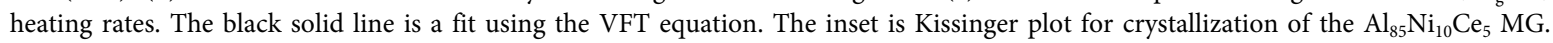


more accurate determination of $m$ based on DSC measurements. In Fig. S1c, we show that for the present $\mathrm{Al}_{85} \mathrm{Ni}_{10} \mathrm{Ce}_{5}$ MG, this method ( $m=125 \pm 3$ ) and ours ( $m=$ $132 \pm 3$ ) yield roughly the same values of fragility within the experimental uncertainty. As another check for the high fragility for the present Al-MG, we used the correlation between thermodynamic properties with fragility: $m \approx m_{\text {cal }}=56 T_{\mathrm{g}} \Delta C_{\mathrm{p}}\left(T_{\mathrm{g}}\right) / \Delta H_{\mathrm{m}}$, where $\Delta C_{\mathrm{p}}\left(T_{\mathrm{g}}\right)$ is the heat capacity jump at $T_{\mathrm{g}}, \Delta H_{\mathrm{m}}$ is the enthalpy of melting. The theory was firstly reported by Wang et al. [58] and subsequently rationalized within framework of the random first order transition. With these parameters measured by FSC (Fig. S1d), we estimate $m_{\text {cal }}=138 \pm 5$ by the above relation, which is in good agreement with the FSC $(m=$ $132 \pm 3$ ). Together, these results suggest that our measurement of fragility by FSC is reliable, and the large $m$ of the present Al-MG could be an inherent feature of the supercooled liquid.

Table 1 lists all the $m$ values studied in this work for 20 typical Al-MGs with different compositions. They all have the similar composition as $\mathrm{Al}_{(100-a-b)} \mathrm{TM}_{a} \mathrm{RE}_{b}$ where $\mathrm{TM}$ is the transition metal $(\mathrm{Co}, \mathrm{Ni}), \mathrm{RE}$ is the rare earth element (Y, La, Ce, Sm). We find that all of them have relatively large $m$ values. Fig. 2 contrasts them with other glass formers with different bonding nature. Specifically, we present the data in an $m-T_{\mathrm{g}}$ plot, since $T_{\mathrm{g}}$ is more likely to be determined by the bonding mechanisms. The Al-MGs are standing out as they have lower $T_{\mathrm{g}}$ but larger $m$. It is noteworthy that several Al-MGs exhibit large fragility values (e.g., $\mathrm{Al}_{86} \mathrm{Ni}_{6.75} \mathrm{Co}_{2.25} \mathrm{Ce}_{5}, m=164 \pm 3$ ) that approach the theoretical upper limit value $m=175 \pm 3$ [41]. For comparisons, the highest fragility in molecular and ionic liquids is $m=145$ for decalin [41] and $m=115$ of $\mathrm{Li}$ acetate $[41,58]$, respectively. And the previously known highest fragility in MGs is $m=136$ of $\mathrm{Ni}_{59.5} \mathrm{Nb}_{40.5}[59,60]$. For polymers [61,62], there are a number of cases where $m>175$, but Sokolov and co-workers [62] have shown there are polymer-specific factors in operation. Thus, we do not include polymers for comparisons. In short, the Al-MGs exhibit the highest $m$ value in MGs and nonpolymeric glasses. Meanwhile, Fig. 2 also suggests that although the values of $T_{\mathrm{g}}$ are concentrated for different types of glass formers, there is no universal correlation between $T_{\mathrm{g}}$ and $m$.

Another interesting feature about the fragilities of the Al-MGs is that they are sensitive to minor compositional adjustments (i.e., micro-alloying). To illustrate this point, Fig. 3 shows four typical systems of Al-MGs by systematically varying the compositions. For example, when replacing $\mathrm{Ni}$ with $\mathrm{Co}$ in the $\mathrm{Al}_{86} \mathrm{Ni}_{9-x} \mathrm{Co}_{x} \mathrm{Ce}_{5} \mathrm{MGs}$, the

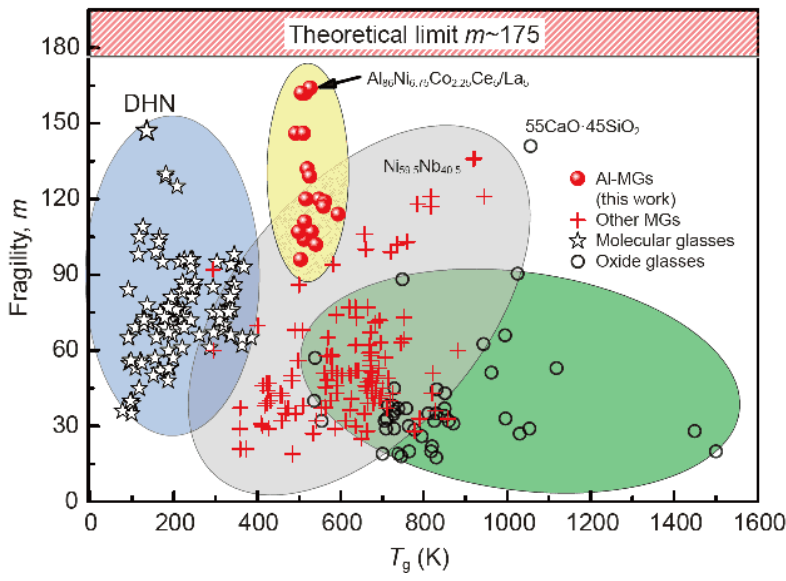

Figure $2 m-T_{\mathrm{g}}$ plot. Glass formers with different bonding nature are compared, including molecular glasses, oxide glasses, the Al-MGs in this work and other MGs.

fragility increase sharply from $m=132 \pm 3$ for $x=0$ to $m=$ $164 \pm 3$ for $x=2$, which means that only $2 \%$ composition variation would remarkably change about $20 \%$ of the magnitude of $m$ values. However, with further increasing of $x$, the $m$ values drop quickly, resulting in a maximum of $m$ value at $x=2$. A qualitatively similar trend is observed in another system with the composition formula $\mathrm{Al}_{86} \mathrm{Ni}_{9-x} \mathrm{Co}_{x} \mathrm{La}_{5}$. Fig. 3c, d illustrate the effects of substitution on fragility for $\mathrm{Ce} / \mathrm{Sm}$ in the $\mathrm{Al}_{85} \mathrm{Ni}_{10} \mathrm{Ce}_{x} \mathrm{Sm}_{5-x}$ MGs and for $\mathrm{Al} / \mathrm{Ni}$ in the $\mathrm{Al}_{95-x} \mathrm{Ni}_{x} \mathrm{Sm}_{5} \mathrm{MGs}$, respectively. Again, they indicate that minor composition changes introduce large variation of $m$.

We next studied the relation between glass forming ability of Al-MGs and the liquid properties. We characterized the glass forming ability of the Al-MGs by the $h_{\mathrm{c}}$ of the ribbons that can retain the fully amorphous phase. As summarized in Table 1, these values range from $10 \mu \mathrm{m}$ $\left(\mathrm{Al}_{90} \mathrm{Ce}_{10}\right)$ to $170 \mu \mathrm{m}\left(\mathrm{Al}_{86} \mathrm{Ni}_{6.75} \mathrm{Co}_{2.25} \mathrm{Y}_{3.25} \mathrm{La}_{1.75}\right)$. We find a modest correlation between $h_{\mathrm{c}}$ and $m$ for Al-based MGs, as shown in Fig. 4a. Specifically, the glass forming ability are characterized by a parameter of $\log \left(d_{\max }^{2}\right)$ as introduced by Johnson et al. [42], where $d_{\max }$ is the critical effective diameter for a rod-like MG. According to Ref. [42], we use $d_{\max }=4 h_{c}$. A general trend can be observed that a large $m$ value could weaken the glass forming ability.

Meanwhile, we include more data from literature [42] about the glass forming ability and plot them against $m$ (Fig. 4a). Thus, it covers nearly the whole range of $m$. One can see that these data exhibit a consistent correlation and the Al-MGs are at the down-right corner: the highest $m$ but the lowest glass forming ability. It is noteworthy that 
for $m<100, \log \left(d_{\max }{ }^{2}\right)$ is nearly a linear function of $m$, but strong deviations (or nonlinear behavior) commences for $m>100$. The data also imply that $m<100$ could be critical for achieving Al-MGs with good glass forming ability. It is noted that the lowest fragility, $m=96$ in our studied Al-MGs, coinciding with the best glass forming ability of the $\mathrm{Al}_{86} \mathrm{Ni}_{6.75} \mathrm{Co}_{2.25} \mathrm{Y}_{3.25} \mathrm{La}_{1.75} \mathrm{MG}$ [30].

Fig. $4 \mathrm{~b}$ plots the $\log \left(d_{\max }^{2}\right)$ versus $T_{\text {rg }}$ for different MGs. One can see that the relation between glass forming ability and $T_{\text {rg }}$ for our Al-MGs is not consistent with other MGs. This means that, unlike other MGs, $T_{\text {rg }}$ may be less effective for controlling the glass forming ability in Al-MGs. Therefore, the present data suggest that the unusual high fragility could be a controlling factor to the marginal glass forming ability of Al-MGs.

We reflect on why the fragilities of Al-MGs are so high and why they tend to correlate with the glass forming ability. At present, the origin of fragility is still not clearly established [40,63-68]. A useful proposal that might be relevant for the Al-MGs can be developed based on the work of Tanaka and coworkers $[67,68]$. They suggested that both the glass formation and fragility were related to the frustration of medium-range crystalline order, which had similar structural features with the crystalline ground state. By simulations of several two-dimensional models, they demonstrated that systems suffering from weaker frustration effects against crystallization were more fragile $[67,68]$. This argument seems to be consistent with our experimental findings on the correlation between fragility and glass forming ability. In general, for MGs the dominant structural features might not be the mediumrange crystalline order, but the icosahedral or five-fold orders, instead [63,69]. However, recently Sun et al. [70] revealed that in an $\mathrm{Al}_{90} \mathrm{Sm}_{10} \mathrm{MG}$, the structural order was indeed similar with the crystalline, suggesting the basic promise (i.e., medium-range crystalline order) of Tanaka's proposal was satisfied in the Al-MGs. Meanwhile, the XRD pattern of the Al-MGs (Figs S10-S12) show low angle diffraction humps indicating the medium range orders (1-2 nm, depending on compositions). In this way, the very high fragility might be the result of weaker structural frustration in Al-MGs, since the $\mathrm{Al}$
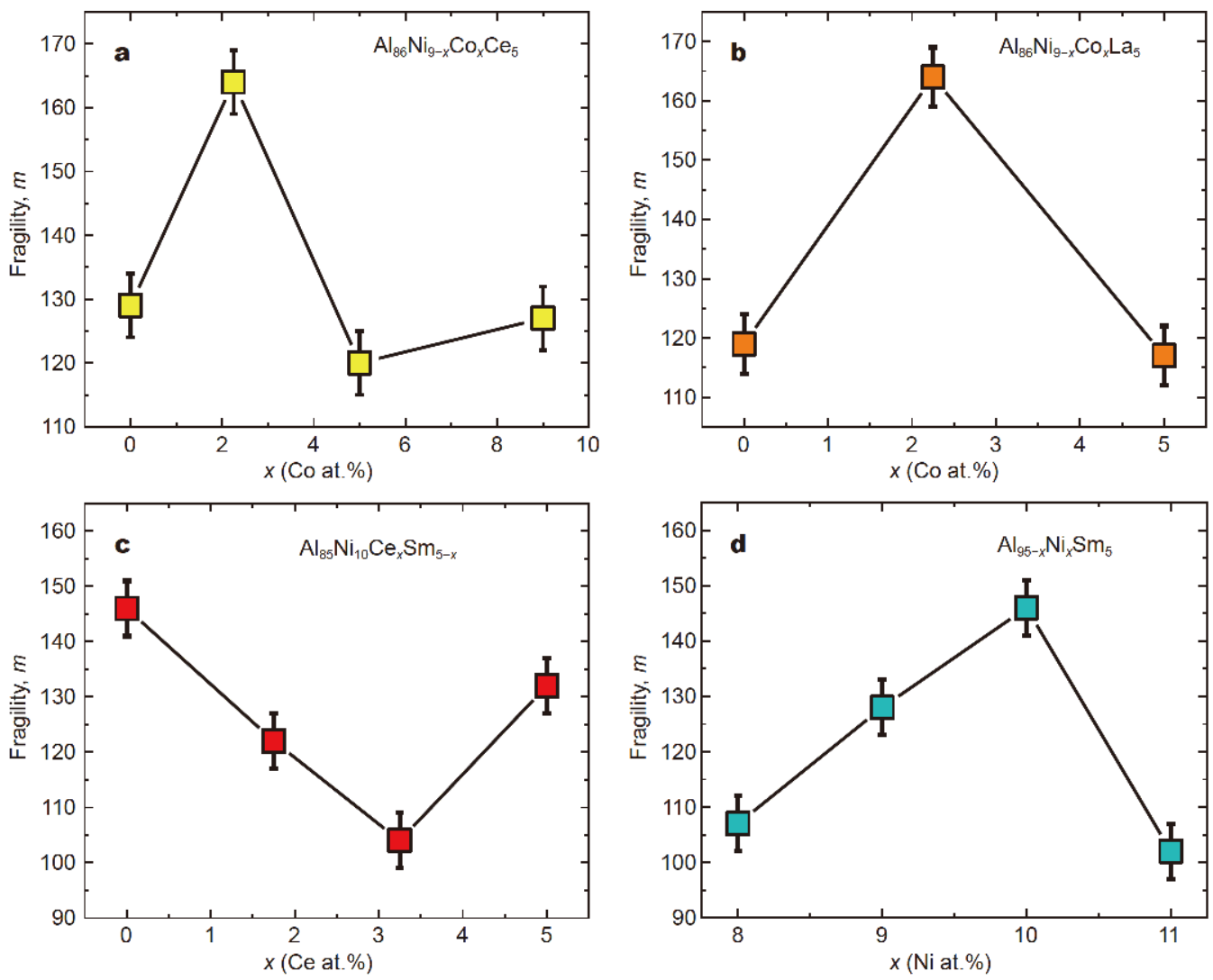

Figure 3 Micro-alloying effects on fragility in different Al-MGs. (a) $\mathrm{Al}_{86} \mathrm{Ni}_{9-x} \mathrm{Co}_{x} \mathrm{Ce}_{5}$ ( $x=0,2.25,5,9$ at.\%); (b) $\mathrm{Al}_{86} \mathrm{Ni}_{9-x} \mathrm{Co}_{x} \mathrm{La}_{5}(x=0,2.25,5$ at.\%); (c) $\mathrm{Al}_{85} \mathrm{Ni}_{10} \mathrm{Ce}_{x} \mathrm{Sm}_{5-x}\left(x=0,1.75,3.25,5\right.$ at.\%); (d) $\mathrm{Al}_{95-x} \mathrm{Ni}_{x} \mathrm{Sm}_{5}(x=8,9,10,11$ at.\%). 

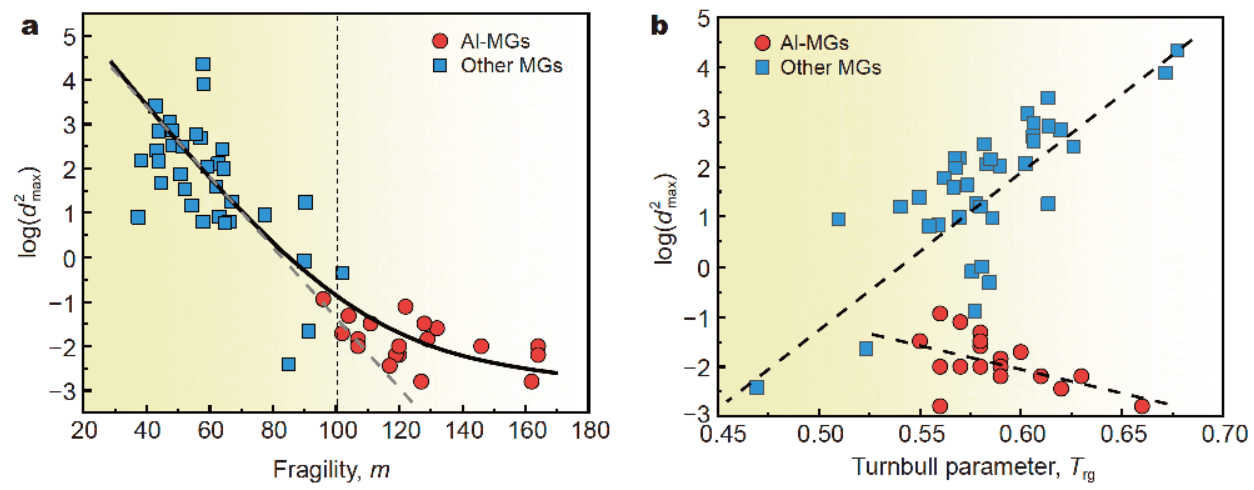

Figure 4 Relationship between glass forming ability and fragility (a), the ratio of the $T_{\mathrm{rg}}$ (b) for Al-MGs. To be consistent with previous works, the $T_{\mathrm{g}}$ are used values at a DSC heating rate of $20 \mathrm{~K} \mathrm{~min}^{-1}$, which is extrapolated from the FSC data. Using $T_{\mathrm{g}}$ based on other heating rate gives the similar results.

element takes up more than $80 \%$.

Another framework to rationalize the fragility is based on the interatomic potentials [65,66]. Krausser et al. [65] proposed that MGs with a steeper (or harder) repulsive part of the interatomic interaction were more fragile. On the other hand, Pueblo et al. [66] suggested reversely: MGs with softer repulsive interaction would be more fragile. As the Al-MGs having exceptional high fragility, they would offer a model system to judge the different views. Accurate deterioration of the inter-atomic potential [69] (e.g., based on density functional theory) would be helpful.

In summary, we have shown that by FSC to suppress the early crystallization, the supercooled liquid state of the Al-MGs can be directly characterized. The results indicate that the Al-MGs are unique as they have very large liquid fragility, with some of them approaching the theoretical upper-limit value. They provide a benchmark model for understanding the origin of fragility and the fragility-property correlation in glasses. We reveal a nonlinear correlation between fragility and the glass forming ability, which provides a useful guide for designing AlMGs and other MGs.

Received 13 June 2019; accepted 23 July 2019;

published online 28 August 2019

1 Johnson WL. Bulk glass-forming metallic alloys: Science and technology. MRS Bull, 1999, 24: 42-56

2 Wang WH. The elastic properties, elastic models and elastic perspectives of metallic glasses. Prog Mater Sci, 2012, 57: 487-656

3 Schroers J, Hodges TM, Kumar G, et al. Thermoplastic blow molding of metals. Mater Today, 2011, 14: 14-19

4 Hirata A, Guan P, Fujita T, et al. Direct observation of local atomic order in a metallic glass. Nat Mater, 2011, 10: 28-33

5 Schroers J. Bulk metallic glasses. Phys Today, 2013, 66: 32-37
6 Liu W, Zhang H, Shi JA, et al. A room-temperature magnetic semiconductor from a ferromagnetic metallic glass. Nat Commun, 2016, 7: 13497

7 Hu F, Zhu S, Chen S, et al. Amorphous metallic NiFeP: A conductive bulk material achieving high activity for oxygen evolution reaction in both alkaline and acidic media. Adv Mater, 2017, 29: 1606570

8 Sarac B, Ivanov YP, Chuvilin A, et al. Origin of large plasticity and multiscale effects in iron-based metallic glasses. Nat Commun, 2018, 9: 1333

9 Zhu F, Song S, Reddy KM, et al. Spatial heterogeneity as the structure feature for structure-property relationship of metallic glasses. Nat Commun, 2018, 9: 3965

10 Yu HB, Wang WH, Samwer K. The $\beta$ relaxation in metallic glasses: An overview. Mater Today, 2013, 16: 183-191

11 Zeng Q, Sheng H, Ding Y, et al. Long-range topological order in metallic glass. Science, 2011, 332: 1404-1406

12 Sun Y, Concustell A, Greer AL. Thermomechanical processing of metallic glasses: Extending the range of the glassy state. Nat Rev Mater, 2016, 1: 16039

13 Hechler S, Ruta B, Stolpe M, et al. Microscopic evidence of the connection between liquid-liquid transition and dynamical crossover in an ultraviscous metallic glass former. Phys Rev Mater, 2018, 2: 085603

14 Giordano VM, Ruta B. Unveiling the structural arrangements responsible for the atomic dynamics in metallic glasses during physical aging. Nat Commun, 2016, 7: 10344

15 Ketkaew J, Chen W, Wang $\mathrm{H}$, et al. Mechanical glass transition revealed by the fracture toughness of metallic glasses. Nat Commun, 2018, 9: 3271

16 Lüttich M, Giordano VM, Le Floch S, et al. Anti-aging in ultrastable metallic glasses. Phys Rev Lett, 2018, 120: 135504

17 Zhao SF, Wang PF, Cheng X, et al. Anomalous low-temperature transport property of oxygen containing high-entropy Ti-Zr-Hf$\mathrm{Cu}-\mathrm{Ni}$ metallic glass thin films. Sci China Mater, 2019, 62: 907-912

18 Greer AL. Metallic glasses...on the threshold. Mater Today, 2009, 12: $14-22$

19 Gross O, Riegler SS, Stolpe M, et al. On the high glass-forming ability of Pt-Cu-Ni/Co-P-based liquids. Acta Mater, 2017, 141: 109-119

20 Frey M, Busch R, Possart W, et al. On the thermodynamics, ki- 
netics, and sub- $T_{\mathrm{g}}$ relaxations of $\mathrm{Mg}$-based bulk metallic glasses. Acta Mater, 2018, 155: 117-127

21 Li MX, Zhao SF, Lu Z, et al. High-temperature bulk metallic glasses developed by combinatorial methods. Nature, 2019, 569: 99-103

22 Li Y, Zhao S, Liu Y, et al. How many bulk metallic glasses are there? ACS Comb Sci, 2017, 19: 687-693

23 Yang BJ, Yao JH, Chao YS, et al. Developing aluminum-based bulk metallic glasses. Philos Mag, 2010, 90: 3215-3231

24 Jiang JZ, Hofmann D, Jarvis DJ, et al. Low-density high-strength bulk metallic glasses and their composites: A review. Adv Eng Mater, 2015, 17: 761-780

25 Suryanarayana C, Inoue A. Iron-based bulk metallic glasses. Int Mater Rev, 2013, 58: 131-166

26 Li HX, Lu ZC, Wang SL, et al. Fe-based bulk metallic glasses: Glass formation, fabrication, properties and applications. Prog Mater Sci, 2019, 103: 235-318

27 Shen Y, Perepezko JH. Al-based amorphous alloys: Glass-forming ability, crystallization behavior and effects of minor alloying additions. J Alloys Compd, 2017, 707: 3-11

$28 \mathrm{Na} \mathrm{JH}$, Demetriou MD, Floyd M, et al. Compositional landscape for glass formation in metal alloys. Proc Natl Acad Sci USA, 2014, 111: 9031-9036

29 He Y, Poon SJ, Shiflet GJ. Synthesis and properties of metallic glasses that contain aluminum. Science, 1988, 241: 1640-1642

30 Wu NC, Zuo L, Wang JQ, et al. Designing aluminum-rich bulk metallic glasses via electronic-structure-guided microalloying. Acta Mater, 2016, 108: 143-151

31 Yang BJ, Lu WY, Zhang JL, et al. Melt fluxing to elevate the forming ability of Al-based bulk metallic glasses. Sci Rep, 2017, 7: 11053

32 Lou HB, Wang XD, Xu F, et al. $73 \mathrm{~mm}$-diameter bulk metallic glass rod by copper mould casting. Appl Phys Lett, 2011, 99: 051910

33 Nishiyama N, Takenaka $\mathrm{K}$, Miura $\mathrm{H}$, et al. The world's biggest glassy alloy ever made. Intermetallics, 2012, 30: 19-24

34 Li Y, Guo Q, Kalb JA, et al. Matching glass-forming ability with the density of the amorphous phase. Science, 2008, 322: 1816-1819

35 Ren F, Ward L, Williams T, et al. Accelerated discovery of metallic glasses through iteration of machine learning and high-throughput experiments. Sci Adv, 2018, 4: eaaq1566

36 Sun Y, Zhang F, Yang L, et al. Effects of dopants on the glass forming ability in Al-based metallic alloy. Phys Rev Mater, 2019, 3: 023404

37 Angell CA. Formation of glasses from liquids and biopolymers. Science, 1995, 267: 1924-1935

38 Orava J, Greer AL, Gholipour B, et al. Characterization of supercooled liquid $\mathrm{Ge}_{2} \mathrm{Sb}_{2} \mathrm{Te}_{5}$ and its crystallization by ultrafast-heating calorimetry. Nat Mater, 2012, 11: 279-283

39 Wei S, Evenson Z, Stolpe M, et al. Breakdown of the Stokes-Einstein relation above the melting temperature in a liquid phasechange material. Sci Adv, 2018, 4: eaat8632

40 Mauro NA, Blodgett M, Johnson ML, et al. A structural signature of liquid fragility. Nat Commun, 2014, 5: 4616

41 Wang LM, Mauro JC. An upper limit to kinetic fragility in glassforming liquids. J Chem Phys, 2011, 134: 044522

42 Johnson WL, Na JH, Demetriou MD. Quantifying the origin of metallic glass formation. Nat Commun, 2016, 7: 10313

43 Zheng Q, Zhang Y, Montazerian M, et al. Understanding glass through differential scanning calorimetry. Chem Rev, 2019, 119: 7848-7939

$44 \mathrm{Hu} \mathrm{L}$, Bian X, Wang W, et al. Liquid fragility and characteristic of the structure corresponding to the prepeak of AlNiCe amorphous alloys. Acta Mater, 2004, 52: 4773-4781

45 Pogatscher S, Leutenegger D, Schawe JEK, et al. Solid-solid phase transitions via melting in metals. Nat Commun, 2016, 7: 11113

46 Wang JQ, Shen Y, Perepezko JH, et al. Increasing the kinetic stability of bulk metallic glasses. Acta Mater, 2016, 104: 25-32

47 Bai FX, Yao JH, Wang YX, et al. Crystallization kinetics of an Aubased metallic glass upon ultrafast heating and cooling. Scripta Mater, 2017, 132: 58-62

48 Zhao B, Yang B, Abyzov AS, et al. Beating homogeneous nucleation and tuning atomic ordering in glass-forming metals by nanocalorimetry. Nano Lett, 2017, 17: 7751-7760

49 Kurtuldu G, Shamlaye KF, Löffler JF. Metastable quasicrystalinduced nucleation in a bulk glass-forming liquid. Proc Natl Acad Sci USA, 2018, 115: 6123-6128

50 Shen Y, Perepezko JH. Investigation of the nucleation delay time in Al-based metallic glasses by high rate calorimetry. J Non-Crystalline Solids, 2018, 502: 9-14

51 Schawe JEK, Löffler JF. Existence of multiple critical cooling rates which generate different types of monolithic metallic glass. Nat Commun, 2019, 10: 1337

52 Spieckermann F, Steffny I, Bian X, et al. Fast and direct determination of fragility in metallic glasses using chip calorimetry. Heliyon, 2019, 5: e01334

53 Gao Y, Zhao B, Vlassak JJ, et al. Nanocalorimetry: Door opened for in situ material characterization under extreme non-equilibrium conditions. Prog Mater Sci, 2019, 104: 53-137

54 Yang B, Schmelzer JWP, Zhao B, et al. Glass transition and primary crystallization of $\mathrm{Al}_{86} \mathrm{Ni}_{6} \mathrm{Y}_{4.5} \mathrm{Co}_{2} \mathrm{La}_{1.5}$ metallic glass at heating rates spanning over six orders of magnitude. Scripta Mater, 2019, 162: $146-150$

55 Gao M, Perepezko JH. Al-based amorphous metallic plastics. Adv Eng Mater, 2019, 21: 1800930

56 Senkov ON. Correlation between fragility and glass-forming ability of metallic alloys. Phys Rev B, 2007, 76: 104202

57 Evenson Z, Gallino I, Busch R. The effect of cooling rates on the apparent fragility of Zr-based bulk metallic glasses. J Appl Phys, 2010, 107: 123529

58 Wang LM, Angell CA, Richert R. Fragility and thermodynamics in nonpolymeric glass-forming liquids. J Chem Phys, 2006, 125: 074505

59 Novikov VN, Sokolov AP. Correlation of fragility and Poisson's ratio: Difference between metallic and nonmetallic glass formers. Phys Rev B, 2006, 74: 064203

60 Mukherjee S, Schroers J, Zhou Z, et al. Viscosity and specific volume of bulk metallic glass-forming alloys and their correlation with glass forming ability. Acta Mater, 2004, 52: 3689-3695

61 Xie SJ, Schweizer KS. Nonuniversal coupling of cage scale hopping and collective elastic distortion as the origin of dynamic fragility diversity in glass-forming polymer liquids. Macromolecules, 2016, 49: 9655-9664

62 Dalle-Ferrier C, Kisliuk A, Hong L, et al. Why many polymers are so fragile: A new perspective. J Chem Phys, 2016, 145: 154901

63 Ding J, Cheng YQ, Sheng H, et al. Short-range structural signature of excess specific heat and fragility of metallic-glass-forming supercooled liquids. Phys Rev B, 2012, 85: 060201

64 Wei S, Stolpe M, Gross O, et al. Linking structure to fragility in bulk metallic glass-forming liquids. Appl Phys Lett, 2015, 106: 181901

65 Krausser J, Samwer KH, Zaccone A. Interatomic repulsion softness 
directly controls the fragility of supercooled metallic melts. Proc Natl Acad Sci USA, 2015, 112: 13762-13767

66 Pueblo CE, Sun M, Kelton KF. Strength of the repulsive part of the interatomic potential determines fragility in metallic liquids. Nat Mater, 2017, 16: 792-796

67 Shintani H, Tanaka H. Frustration on the way to crystallization in glass. Nat Phys, 2006, 2: 200-206

68 Kawasaki T, Araki T, Tanaka H. Correlation between dynamic heterogeneity and medium-range order in two-dimensional glassforming liquids. Phys Rev Lett, 2007, 99: 215701

69 Sheng HW, Cheng YQ, Lee PL, et al. Atomic packing in multicomponent aluminum-based metallic glasses. Acta Mater, 2008, 56: 6264-6272

70 Sun Y, Zhang F, Ye Z, et al. 'Crystal genes' in metallic liquids and glasses. Sci Rep, 2016, 6: 23734

Acknowledgements Discussions with Dr. Shi-Yu Liu and Dr. Yang Sun are appreciated. We thank the supports from the National Thousand Young Talents Program of China.

Author contributions Yu HB directed the research. Yang Q conducted the experiments. All authors contributed to experimental design, data analysis, and interpretation. Yang Q and Yu HB wrote the manuscript.

Conflict of interest The authors declare that they have no conflict of interest.

Supplementary information Supporting materials are available in the online version of the paper.

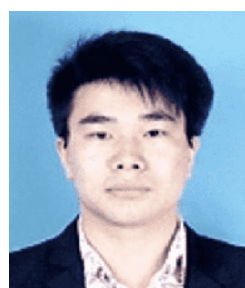

Qun Yang received his Bachelor's degree from Fuyang Normal University in 2016 and is pursuing $\mathrm{PhD}$ degree at Huazhong University of Science and Technology (HUST). His research interest mainly focuses on the relaxation of glass materials, the properties of glass formers and the nature of glass transition.

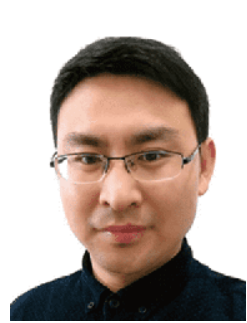

Hai-Bin Yu is currently a professor at HUST. His research interest mainly focuses on the physical properties of metallic glasses and the relaxation dynamics of disordered systems.

\section{超快扫描量热揭示铝基金属玻璃过冷液态: 接近 液体脆度的理论极限}

杨群 $^{1}$, 黄靖 ${ }^{2}$, 秦晓慧 ${ }^{1}$ 葛法熙 ${ }^{1}$, 于海滨 $^{{ }^{*}}$

摘要 铝基非晶合金具有密度低、强度高、耐腐蚀等诸多优异性 能; 然而, 铝基非晶合金形成能力差, 一般需要非常高的冷却速率, 这限制了铝基非晶合金的应用. 玻璃形成理论认为形成能力与过 冷液体密切相关. 但在一般升温测量时, 铝基非晶合金不显示玻璃 转变或过冷液体, 而是直接变成晶态. 目前为止, 关于铝基非晶合金 的玻璃转变和过冷液体属性仍然是未知的. 本文采用超快速差热 分析方法(Flash DSC)使得升温速度达到 $10000 \mathrm{~K} \mathrm{~s}^{-1}$, 测量了 20 余 种常见铝基非晶合金的玻璃转变行为和过冷液体特征. 发现铝基 非晶合金普遍具有很高的液体脆度系数 $(m)$, 其中某些成分 $m>160$, 已经接近理论上预测的脆度系数上限 $m \sim 175$. 通过系统研究这些成 分的形成能力, 发现铝基非晶合金的玻璃形成能力与脆度系数成 反相关, 而且这种相关不是线性的. 只有 $m<100$ 时, 降低 $m$ 才会对玻 璃形成能力有明显影响; 相反, $m>100$ 的玻璃形成力普遍较弱, 而且 随 $m$ 变化不显著. 因此, 过高的液体脆度系数可能是铝基非晶合金 形成能力差的一个重要原因. 